\title{
Anniversary issues
}

\author{
March and April of this year mark the anniversary of a number of key discoveries in the history \\ of nanotechnology.
}

In March 1986, the atomic force microscope (AFM) was introduced by Gerd Binnig, Calvin Quate and Christoph Gerber with a paper in the journal Physical Review Letters titled simply 'Atomic force microscope'. This was 5 years (to the month) after the precursor to the AFM, the scanning tunnelling microscope (STM), had first been successfully tested at IBM's Zurich Research Laboratory by Binnig and the late Heinrich Rohrer, and 7 months before Binnig and Rohrer were awarded a share of the Nobel Prize in Physics for the design of the STM (the prize was shared with Ernst Ruska, the inventor of the electron microscope). Achieving atomic resolution with the AFM proved more difficult than with the STM. It was, for example, only two years after its invention that the STM provided atomic-resolution images of an icon of surface science, the $7 \times 7$ surface reconstruction of $\mathrm{Si}(111)$ (ref. 2), whereas it took 8 years to achieve a similar feat with the $\mathrm{AFM}^{3,4}$. But the versatility and inherent capabilities of the AFM mean that, 30 years on from its invention, it is now a standard method of a modern nanotech lab.

The AFM works by scanning a sharp tip attached to a flexible cantilever across a sample while measuring the interaction between the tip and the sample surface. The technique can operate in a range of environments, including in liquid and in air, and unlike the STM, it can be used with insulating materials; in their original paper, Binnig and colleagues used the instrument to analyse an aluminium oxide sample. At first, the AFM was operated in 'contact mode', in which the tip scans a sample while in close contact with the surface, but alternative schemes quickly appeared. In particular, 'non-contact mode', in which the cantilever sits a small distance above the surface and is oscillated close to its resonant frequency, appeared a year after the AFM debuted 5 , and 'tapping mode', in which the cantilever is oscillated at its resonant frequency and gently taps the sample as it scans, followed a few years later ${ }^{6}$. These technological advances were key to the emergence of the AFM as a force in biological research and technical advances continue to push the possibilities of the instrument today.

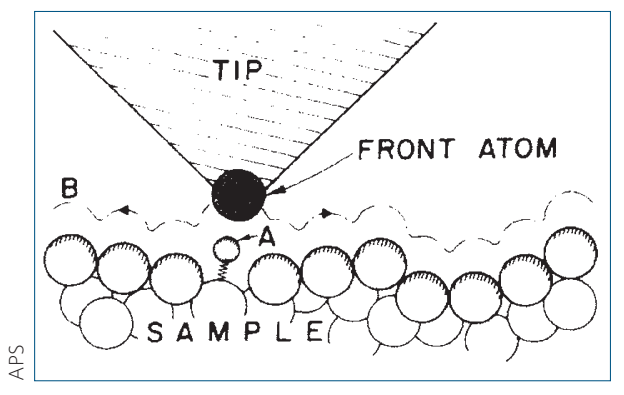

Schematic of the principle of the AFM from the original paper by Binnig and colleagues'.

This continuing evolution can be seen in the field of high-speed atomic force microscopy. Here, by increasing the scan speed of the instrument, the dynamic behaviour of various species, including biomolecules, can be studied. The approach has, for example, been used to image the motor protein myosin $\mathrm{V}$ as it walks along an actin $\operatorname{track}^{7}$ and to examine the kinetics of individual bacterial cell death ${ }^{8}$. Such achievements have been possible due to the miniaturization of silicon and silicon nitride cantilevers. Whereas this approach has proved successful when scanning in liquids, achieving similar imaging speeds in air is difficult. Earlier this year though it was shown that high-speed imaging in air is possible by using cantilevers made from polymers?.

Another branch of atomic force microscopy that also continues to progress is multifrequency imaging ${ }^{10}$. Here, the instrument's probe is excited and/or detected at two or more frequencies. This can lead to improvements in sensitivity and resolution, and the technique has been used to image the subsurface of cells and to map the flexibility of proteins. The AFM has come a long way in 30 years, and there is no sign its development is finished yet. It is not though the only area of nanotechnology celebrating a recent anniversary.

The history of structural DNA nanotechnology can, like the AFM, be traced back to the early 1980s, when Nadrian Seeman suggested that the exquisite base-pairing rules of DNA could be exploited to build artificial selfassembled structures ${ }^{11}$. But the founding experiment of the field came later. In April 1991, Seeman and Junghuei Chen reported building a cube-like molecular complex from DNA using a combination of branched junctions and singlestranded 'sticky' ends ${ }^{12}$. A range of significant advances soon followed, from 2D DNA arrays to DNA-based nanomechanical devices.

Then, in March 2006, the field of structural DNA nanotechnology experienced another decisive moment: Paul Rothemund reported the development of DNA origami ${ }^{13}$. This technique involves folding a long single strand of DNA into a predetermined shape with the help of short 'staple' strands. Used at first to create 2D structures, which were incidentally characterized using the AFM, the approach was quickly expanded to the building of intricate 3D structures and the organization of other species such as nanoparticles and proteins. Twenty-five and ten years on from these discoveries, the drive to improve the structural control of such methods continues $^{14}$, but the search for potential applications is of increasing importance, with applications at the interface with biology of particular interest ${ }^{15}$.

And while on the topic of anniversaries, it should be noted that a certain Nature research journal dedicated to nanoscience and nanotechnology will shortly celebrate its own 10-year anniversary. But that's a story for October.

\footnotetext{
References

1. Binnig, G., Quate, C. F. \& Gerber, Ch. Phys. Rev. Lett. 56, 930-933 (1986)

2. Binnig, G., Rohrer, H., Gerber, Ch. \& Weibel, E. Phys. Rev. Lett. 50, 120-123 (1983)

3. Giessibl, F. J. Science 267, 68-71 (1995).

4. Giessibl, F. J. Mater. Today 8, 32-41 (May, 2005).

5. Martin, Y., Williams, C. C. \& Wickramasinghe, H. K. J. Appl. Phys. 61, 4723-4729 (1987).

6. Zhong, Q., Inniss, D., Kjoller, K. \& Elings, V. B. Surf. Sci. 290, L688-L692 (1993).

7. Kodera, N., Yamamoto, D., Ishikawa, R. \& Ando, T. Nature 468, 72-76 (2010).

8. Fantner, G. E., Barbero, R. J., Gray, D. S. \& Belcher, A. M. Nature Nanotech. 5, 280-285 (2010).

9. Adams, J. D. et al. Nature Nanotech. 11, 147-151 (2016)

10. Garcia, R. \& Herruzo, E. T. Nature Nanotech. 7, 217-226 (2012).

11. Seeman, N. C. J. Theor. Biol. 99, 237-247 (1982).

12. Chen, J. H. \& Seeman, N. C. Nature 350, 631-633 (1991).

13. Rothemund, P. W. K. Nature 440, 297-302 (2006).

14. Zhang, F. et al. Nature Nanotech. 10, 779-784 (2015)

15. Chen, Y.-J., Groves, B., Muscat, R. A. \& Seelig, G.

Nature Nanotech. 10, 748-760 (2015).
} 